Methods One thousand four hundred and six consecutive patients, who underwent CAG at Daxing hospital from February 2007 through to March 2010 were enrolled. Of the 1406 patients, 351 patients were diagnosed as type 2 diabetes mellitus, 1055 patients were diagnosed as non-diabetic mellitus after admission to hospital. By evaluating the coronary angiogram, the patients were not diagnosed to have coronary heart disease (CHD) with less than $50 \%$ diameter stenosis of coronary artery; $\mathrm{CHD}$ was defined as narrowing of the appropriate lumen of $\geq 50 \%$; the procedure of percutaneous coronary intervention (PCI) were performed in the patients with more than or equal to $70 \%$ stenosis; the coronary aortic bypass graft (CABG) surgery had been proposed in patients with left main coronary artery lesions, left main equivalent, diffuse triple coronary artery lesions, two-vessel disease with significant proximal left anterior descending $\mathrm{CAD}$, however the determinations of the therapeutic choice were combined with clinical data.

Results The baseline characteristics of patients with and without diabetics undergone coronary angiography were as following. The age was significantly older in patients with diabetes than without diabetes $(60.22 \pm 9.70$ vs $57.76 \pm 9.94, \mathrm{p}<0.0005)$. More femal patients in the diabetes group than non-diabetes group $(45.30 \%$ vs $35.55 \%, \mathrm{p}<0.0005)$. The morbidity rate of UAP $(64.96 \%$ and $49.86 \%, \mathrm{p}<0.0005)$, and Hypertension $(80.06 \%$ and $69.57 \%$, $\mathrm{p}<0.0005)$ were significantly higher in patients with diabetes than without diabetes. By evaluating the coronary angiogram, more patients were diagnosed to $\mathrm{CHD}$ in the diabetes group than in the non-diabetic group $(92.59 \%$ vs $79.43 \%, \mathrm{p}<0.0005)$; the proportion of the population of the patients with CHD not indicated for PCI was almost identical in the two groups ( $23.30 \%$ vs $25.97 \%, p=0.33)$; the proportion of the patients with $\mathrm{CHD}$ performed the procedure of stent implantation (including the patients receiving follow-up coronary angiography after stenting) were not differ significantly between the two groups ( $35.61 \%$ vs $32.61 \%, p=0.30$ ); more patients with CHD were proposed to perform the CABG in the diabetes group than in the non-diabetic group $(31.91 \%$ vs $17.35 \%$, $\mathrm{p}<0.0005)$.

Conclusion The morbidity rate of coronary heart disease among patients with type 2 diabetes is greater than non-diabetes, patients with type 2 diabetes have a significantly higher rate of coronary artery bypass grafting which had been proposed.

\section{e0467 THE IMPACT OF DIABETES ON LONG TERM FOLLOW UP OF THE PATIENTS WITH CHRONIC TOTAL OCCLUSION POST PERCUTANEOUS INTERVENTION}

doi:10.1136/hrt.2010.208967.467

Liu Wei, Wagatsuma Kenji. Toho University Omori Hospital

The prognosis of patients with chronic total occlusion (CTO) and diabetes mellitus treated with percutaneous coronary intervention (PCI) is not well known.

Methods From Jan 2001 to April 2009, 105 cases of CTO successfully treated with PCI were included. 31 patients with diabetes and 74 without diabetes were compared for angiographic and clinical outcomes (mean follow up $36 \pm 21$ month). Death, myocardial infarction and repeat PCI or coronary artery bypass surgery were considered as a combined primary endpoint.

Results 25 diabetes patients $(78 \%)$ and 67 non-diabetic patients $(89 \%)$ were treated by drug eluting stent $(p=0.37)$. The primary endpoint occurred in $22 \%(n=7)$ of diabetes patients, $10.8 \%(n=8)$ of the patients without diabetes $(p=0.059$; Log rank test), Cox regression showed patients with diabetic group and moderately or severely reduced renal impairment had significant increased risk for MACE (HR: 6.34, 95\% CI 2.06 to 19.56, p<0.001).
Conclusions Our study showed patients with CTO and diabetes have a tendency of poor prognosis after PCI, which may be largely due to the complicated renal impairment.

\section{C0468 EFFECT OF DIFFERENT LOADING DOSES OF ATORVASTATIN ON PERCUTANEOUS CORONARY INTERVENTION FOR ACUTE CORONARY SYNDROMES}

doi:10.1136/hrt.2010.208967.468

Sun Yujiao, Qi Guoxian, Gao Yuan, Zhang Haishan, Pang Xuefeng, Zhao Weihua, Zhang Zixin. Department of Cardiology, The First Affiliated Hospital of China Medical University

Background Percutaneous coronary intervention (PCI)-induced myocardial damage is a major cause of late cardiovascular events. Treatment with atorvastatin before PCI can reduce myocardial damage during the peri-PCI period. Objectives: To compare the safety and myocardial effects of different atorvastatin loading doses and dosing frequency before PCI in non-ST-segment elevation acute coronary syndrome (NSTE-ACS) patients.

Methods 80 NSTE-ACS patients were randomly divided into four groups (20 patients/group). The control group was given $40 \mathrm{mg}$ atorvastatin each night. The three loading dose groups were treated as in the control group, but were given $80 \mathrm{mg}$ atorvastatin $12 \mathrm{~h}$ before PCI (low-load group) in combination with $40 \mathrm{mg}$ atorvastatin $2-4 \mathrm{~h}$ before PCI (mid-load group) or $60 \mathrm{mg}$ atorvastatin $2-4 \mathrm{~h}$ before PCI (high-load group). All patients underwent PCI within 48-72 h of admission, and received $40 \mathrm{mg}$ atorvastatin for at least 1 month after PCI. Changes in myocardial markers and high sensitive C-reactive protein (hs-CRP) were analysed. Patients were followed-up for 30 days to monitor the incidence of major adverse cardiac events (MACE)

Results No deaths or revascularisations were recorded. The incidences of MACE differed significantly between the four groups $(40 \%, 25 \%, 10 \%$ and $0 \%$, respectively, $\mathrm{p}<0.05)$. The incidence of MACE and cardiac troponin I (cTnI) level above the normal range, and post-PCI increases in creatine kinase-myocardial bank (CK-MB) and hs-CRP were significantly higher in the control group than in the high-load group (all, $\mathrm{p}<0.007$ ). The post-PCI cTnI and CK-MB levels were similar to the pre-PCI levels in the high-load group, but increased significantly in the control and low-load groups. The magnitude of the hs-CRP level increased was significantly lower in the high-load group than in the control and low-load groups. The post-PCI alanine aminotransferase levels in all four groups were significantly higher than the pre-PCI levels, but were within normal ranges. No myalgia or myasthenia was observed.

Conclusion This study shows that short-term atorvastatin loading before PCI was well tolerated had beneficial myocardial effects in patients with NSTE-ACS.

\section{e0469 MULTIMODALITY IMAGING EVALUATION OF FUNCTIONAL AND CLINICAL BENEFITS OF PERCUTANEOUS CORONARY INTERVENSION ON PATIENTS WITH CHRONIC TOTAL OCCLUSION LESION}

doi:10.1136/hrt.2010.208967.469

Dongdong Sun, Yue Tian, Jing Wang, Zhu Shunming, Yi Huan, Wejie Li, Feng Cao. Department of Cardiovascular Medicine, Radiology \& Nulclear Medicine, Xijing Hospital, Fourth Military Medical University, China

Aims To determine the effects of percutaneous coronary intervension (PCI) on cardiac perfusion, cardiac function and quality of life in patients with chronic total occlusion (CTO) lesion in left anterior descending (LAD) coronary artery. 